\title{
Nanopartículas de plata en el tratamiento de la micosis asociada al pie diabético
}

Silver nanoparticles to treat mycosis associated with diabetic foot Nanopartículas de prata no tratamento da micose associada ao pé diabético

Pablo Orellano¹, María del Huerto Urdangarin², Jenifer Silva³, Germán Botto4, Analía González ${ }^{5}$, Lilián Vucovich ${ }^{6}$, Vivian Peirano ${ }^{7}$

\section{Resumen}

Una proporción importante de infecciones en el pie diabético consisten en onicomicosis y tinea pedis, problema común en el pie, amenazante de la viabilidad del tejido que puede provocar infecciones bacterianas secundarias. Requieren períodos prolongados de tratamiento antimicótico con alta tasas de recaídas y reinfección. Diversos estudios han mostrado la seguridad y eficacia de las nanopartículas de plata (NP Ag) como agente antimicrobiano. Realizamos un estudio donde se evaluó el tratamiento con NP Ag en dermatomicosis del pie de pacientes diabéticos.

Método: estudio piloto, abierto, prospectivo, randomizado y controlado en pacientes que asisten a una policlínica de pie diabético. Dieciocho pacientes cumplieron con los criterios de inclusión conformándose dos grupos homogéneos. Ambos grupos recibieron tratamiento estándar que consistió en antimicótico tópico y desbastado mecánico. El grupo intervención utilizó un textil (medias) confeccionadas con hilos con NP Ag. Se realizó control clínico y microbiológico hasta las 12 semanas. Se evaluó el porcentaje de remisión y el tiempo hasta alcanzar el mismo.

Resultados: predominó la onicomicosis y el germen Trichophyton rubrum. En el grupo intervención se logró un mayor porcentaje de remisión de lesiones y en un tiempo menor que el grupo control.

Conclusiones: el uso de medias confeccionadas con hilos de NP Ag se asoció con una mayor probabilidad de curación completa en un período de 12 semanas a pesar de que el número de pacientes no permitió llegar al nivel de significación estadística, pudiendo contribuir a la prevención de infecciones o úlceras suplementarias en el pie diabético.

\begin{tabular}{|c|c|c|c|}
\hline Palabras clave: & $\begin{array}{l}\text { Nanopartículas de plata } \\
\text { Onicomicosis } \\
\text { Antifúngicos } \\
\text { Pie diabético }\end{array}$ & Key words: & $\begin{array}{l}\text { Silver nanoparticles } \\
\text { Onychomycosis } \\
\text { Antifungal agents } \\
\text { Diabetic foot }\end{array}$ \\
\hline
\end{tabular}

\footnotetext{
1. Ex Profesor Adjunto Endocrinología y Metabolismo, Unidad de Pie Diabético, Hospital de Clínicas, Facultad de Medicina. Universidad de la República. Uruguay.

2. Podóloga del Servicio de Endocrinología. Unidad de Pie Diabético, Hospital Regional Mercedes. Soriano. Uruguay.

3. Asistente de Endocrinología y Metabolismo, Facultad de Medicina, Universidad de la República. Uruguay.

4. Profesor Adjunto Departamento de Métodos Cuantitativos, Facultad de Medicina, Universidad de la República. Uruguay. PhD Candidate, Department of Microbiology and Immunology, Montana State University.

5. Licenciada en Enfermería. Servicio de Endocrinología. Unidad de Pie Diabético, Hospital Regional Mercedes. Soriano. Uruguay.

6. Prof. Agdo. Técn. Directora de la Carrera de Podología EUTM, Unidad de Pie Diabético, Facultad de Medicina, Universidad de la República, Uruguay.

7. Magíster Licenciada en Laboratorio. Microbiología, Hospital Regional Mercedes. Soriano. Uruguay.

Unidad de Pie Diabético. Escuela de Tecnología Médica, Carrera de Podología, Hospital de Clínicas. Montevideo. Servicio de Endocrinología Hospital Zoilo A Chelle. Mercedes. Uruguay.

Correspondencia: Dr. Pablo Orellano. Correo electrónico: porellanoc@gmail.com

El textil con nanopartículas de plata fue proporcionado por la empresa Prumer Ltda.

Los autores declaran no tener conflictos de intereses ni relación financiera con la empresa.

Recibido: $20 / 8 / 20$

Aprobado: $18 / 11 / 20$

Attribution-NonCommercial 4.0 International (CC BY-NC 4.0)
} 


\section{Introducción}

Más de 150 millones de personas en todo el mundo sufren de diabetes, 22 millones en Estados Unidos ${ }^{(1)}$. En Uruguay, la prevalencia estimada ajustada por edad es de 6,8\%-7,5\%, tomando en cuenta las publicaciones de los últimos 30 años ${ }^{(2)}$. Los pacientes con diabetes corren el riesgo de desarrollar una úlcera del pie diabético (UPD) de diverso grado y más del $15 \%$ realmente desarrollará tales úlceras ${ }^{(3)}$. Entre $14 \%$ y $24 \%$ de dichos pacientes requiere amputación.

Luego de una amputación mayor, la sobrevida del paciente disminuye hasta un $50 \%$ a los cinco años, lo cual implica una mortalidad más alta que la de varios tipos de cáncer ${ }^{(4-6)}$. La prevención de la UPD es su mejor tratamiento. Diversos factores de riesgo y desencadenantes de UPD pueden modificarse. La infección por hongos, en particular, aunque relativamente poco visible, es un problema muy común en el pie, si no se trata puede amenazar la viabilidad del tejido en el pie del diabético, provocar una infección bacteriana secundaria y celulitis $^{(7,8)}$

Las dermatofitosis son afecciones de gran importancia por su elevada incidencia, comunes al hombre y a los animales. En el género Trichophyton las especies con mayor incidencia a nivel universal son T. rubrum y $T$. mentagrophytes var. interdigitalis, responsables principales de las tiñas del pie (pie de atleta, tinea pedis) y de la tiña de las uñas (tinea unguium $)^{(9)}$. Las infecciones fúngicas invasivas también pueden ser un patógeno causante de infecciones de tejidos profundos ${ }^{(10)}$. Sigue siendo importante, como iniciativa de salud pública, tratar estas heridas de manera rápida y efectiva.

La mayoría de las modalidades de tratamiento antimicótico requerirán un uso a largo plazo (3 a 9 meses) para ser efectivas ${ }^{(11)}$. El manejo de esta enfermedad a menudo se considera difícil debido a las altas tasas de recaída y reinfección. Los tratamientos tópicos son efectivos si se usan solos para la afectación ungueal leve a moderada. El tratamiento mecánico es efectivo como complemento de la terapia tópica. La terapia sistémica requiere la consideración de los efectos secundarios y el control por parte del paciente y el médico antes de su aplicación. Esto se debe a que los tratamientos logran una concentración efectiva pero temporal del fármaco en los sitios de infección. El enfoque terapéutico ideal debería introducir los fármacos antimicóticos directamente en los sitios de infección, con una liberación controlada y manteniendo una concentración efectiva durante un tiempo prolongado, con un nivel mínimo de respuesta inflamatoria en el sitio de la lesión.

La plata (Ag) ha sido utilizada desde la Antigüedad como biocida. La producción de nanopartículas de plata
(NP Ag) se practica desde hace más de 100 años, siendo uno de los nanomateriales más comúnmente utiliza$\operatorname{dos}^{(12)}$. Con un tamaño entre 1 y $100 \mathrm{~nm}$ en al menos una dimensión, determinan un aumento del radio entre el área de superficie y volumen en que actúa, confiriéndoles modificaciones significativas en sus propiedades físicas, químicas y biológicas útiles en la aplicación de la nanomedicina.

Su tamaño es similar a entidades biológicas, como proteínas (enzimas o receptores) y moléculas de ácidos nucleicos, por lo que podrían interactuar con estas biomoléculas permitiendo modificar su comportamiento o sus propiedades bioquímicas ${ }^{(13)}$.

Estudios preclínicos han documentado la seguridad y eficacia de las NP Ag en el tratamiento antimicrobia no ${ }^{(14-16)}$

$\mathrm{Su}$ desarrollo en textiles funcionales para medicina se nutre de diferentes disciplinas, tales como la electrónica, la ciencia de los polímeros, la nanociencia y la nanotecnología ${ }^{(17,18)}$. El antimicrobiano utilizado se une al grupo funcional de la fibra de forma que su liberación es controlada por la humedad del ambiente, o por enlaces químicos que lo liberan al interactuar con el microorganismo, esto le confiere diferentes grados de solidez al lavado y de acción biocida. Diversos tipos de fibras tratadas con NP Ag han probado actividad antimicrobiana contra $E$. coli, S. aureus, $K$. pneumoniae y especies de Streptococcus ${ }^{(19-23)}$. La actividad antibacteriana y antifúngica del textil se evalúan bajo una serie de normas. El hongo más evaluado ha sido $A$. niger ${ }^{(24)}$.

\section{Objetivo}

Nuestro estudio evalúa el uso de las NP Ag vehiculizadas en un textil como una forma de liberación controlada dérmica para el tratamiento complementario de las dermato y onicomicosis en pacientes que asistieron a una policlínica de pie diabético entre 2018 y 2020.

Objetivo específico: comparar el tiempo de remisión de la lesión micótica entre grupos con tratamiento estándar y el agregado de NP Ag.

\section{Material y método}

Estudio piloto, abierto, prospectivo, randomizado y controlado de 12 semanas de duración. Un autor del estudio generó la secuencia aleatoria usando Excel (Microsoft Corporation, Redmond, WA). La selección no dependió de la demografía de los pacientes o de los sitios de las lesiones. Se evaluaron 18 pacientes diabéticos que concurrieron a la policlínica de tratamiento del pie y que cumplieron con los criterios de inclusión y exclusión para la elegibilidad del estudio. 


\section{Criterios de inclusión}

Diabético tipo 1 o 2, entre 18-85 años. Paciente que concurre a la Policlínica de Pie Diabético. Pacientes con micosis ungueal, interdigital o plantar no sobreinfectada. Paciente que firma el consentimiento informado.

\section{Criterios de exclusión}

Micosis interdigital o plantar sobreinfectada. Úlcera infectada. Patología psiquiátrica mayor. Retinopatía diabética severa. Alergia a los productos con plata. Imposibilidad de concurrir a los controles. Embarazo.

El tratamiento estándar para ambos grupos consistió en el uso de antimicótico tópico para la micosis interdigital plantar y para la afectación ungueal leve a moderada junto a tratamiento mecánico como complemento de la terapia tópica, que incluyeron la avulsión y la abrasión de las uñas, en especial en la onicomicosis subungueal $\operatorname{parcial}^{(25)}$.

Al grupo intervención se le adicionó el uso durante 12 semanas de medias confeccionadas con hilos de NP Ag, alentando a los pacientes a usarlas diariamente.

Dos expertos evaluaron clínicamente una vez por semana a todos los pacientes, se encargaron de la obtención de las muestras microbiológicas y del registro fotográfico de las lesiones. Se evaluó la remisión clínica y microbiológica, y el tiempo hasta su remisión.

Se recabaron los siguientes datos: edad, sexo, fármacos que recibían los pacientes, años del diagnóstico de diabetes y datos antropométricos. Se evaluó el pulso en miembros inferiores y se calculó el índice tobillo/brazo (T/B) utilizando un equipo Doppler portátil. Se realizó evaluación bioquímica metabólica. La muestra microbiológica se recolectó con bisturí y se envió al laboratorio de microbiología. El aislamiento del hongo se realizó en medios selectivos y su identificación por métodos micológicos estándar ${ }^{(26,27)}$.

\section{Características del material con nanopartículas de plata a utilizar}

Se trata de medias confeccionadas con hilo al que se le incorporó NP Ag de 20-40 nm aplicada a una concentración del $2 \%$ mediante técnica de presión electro estática que permite incorporar el nanocompuesto en la molécula.

La identificación y cuantificación del elemento Ag y los compuestos presentes en el hilo fueron realizadas en el departamento de la Unidad de Microscopía Electrónica de Barrido (UMEB) de la Facultad de Ciencias. La evaluación técnica (análisis de agua) del hilo con NP Ag mostró que la incorporación del nanoproducto en el interior del hilo permite una adherencia que garantiza 15 ciclos de lavado manteniendo la eficacia antimicrobiana. Su eficacia bactericida se evaluó con el método basado en AATCC 100-2012 ${ }^{(24)}$. Los participantes fueron informados sobre las características del estudio y firmaron el consentimiento. El estudio fue aprobado por el Comité de Ética del Hospital de Clínicas.

\section{Análisis estadístico}

La elaboración del análisis estadístico se realizó en $\mathrm{R}$ 3.6.2 $2^{(28)}$. Las distribuciones de variables continuas (edad, duración de la enfermedad, índice de masa corporal, etc.) se compararon utilizando test de Student o de Wilcoxon. Para las variables dicotómicas (cierre de úlceras, desaparición de lesiones, etc.) se utilizaron las pruebas de chi cuadrado o Fisher ${ }^{(29)}$. La selección de la prueba adecuada en cada caso se basó en el análisis exploratorio de las variables. Las asociaciones entre el tratamiento y la remisión clínica o microbiológica se evaluaron mediante riesgos relativos (evaluando la probabilidad de remisión en el grupo tratado en relación con el grupo control). La significación estadística se calculó a partir de una distribución empírica de valores obtenidos mediante aleatorización. Los tiempos de remisión se analizaron mediante curvas de sobrevida de Kaplan-Meier. Las curvas estimadas para los grupos tratamiento y control se compararon utilizando prueba de Log-rank ${ }^{(30)}$, usando el paquete Survival ${ }^{(31)}$. Se enmascaró el análisis eliminando la información del tratamiento en cada grupo de la base de datos.

\section{Resultados}

Se incluyeron 18 pacientes, 8 pacientes se asignaron al uso del textil, 10 pacientes al grupo control, en este último un paciente abandonó el seguimiento.

Ambos grupos fueron homogéneos en la evaluación demográfica, metabólica, clínica y antropométrica. No hubo diferencias significativas entre ambos grupos (tabla 1).

Predominó la lesión ungueal junto al germen Trichophyton rubrum y en segundo lugar tiña pedis y el germen Trichophyton mentagrophytes.

En la evaluación del resultado primario a los 90 días, el $87,5 \%$ en el grupo de tratamiento con el textil se curó en comparación al $66,7 \%$ en el grupo de tratamiento control $(\mathrm{RR}=1,31$, $\mathrm{p}$-value=0,26). De igual forma, en la remisión microbiológica a los 90 días el 87,5\% en el grupo que utilizó NP Ag curó frente a 55,5\% en el grupo control $(\mathrm{RR}=1,58, \mathrm{p}$-value=0,09). Estas diferencias no alcanzaron la significación estadística (Fisher test, p-value $=0,58$ para remisión clínica, ni en la remisión microbiológica Fisher test, p-value=0,29).

En los tiempos medios para sanar (dentro de 90 días), cuando se evalúa el tiempo a la remisión clínica, observamos una tendencia no significativa (Log-rank 
Tabla 1. Comparación de las variables demográficas, glucémico-metabólicas microbiológicas medidas al inicio del estudio entre ambos grupos.

\begin{tabular}{lcccc}
\hline Variable & Grupo control $(n=10)$ & Grupo textil $(n=8)$ & Valor $p$ & Prueba utilizada \\
\hline Edad (años) & $60,6 \pm 13,8$ & $64,9 \pm 8,2$ & 0,43 & Test t Student \\
Sexo (Mas:Fem) & $5: 5$ & $5: 3$ & 0,66 & Test de Fisher \\
Diagnóstico de DM (años) & $7,1 \pm 4,2$ & $11,4 \pm 5,9$ & 0,10 & Test de Wilcoxon \\
H1Ac (\%) & $7,6 \pm 1,0$ & $9,3 \pm 1,6$ & 0,03 & Test de Wilcoxon \\
IMC (kg/m²) & $28,24,0$ & $26,82,6$ & 0,96 & Test de Wilcoxon \\
Neuropatía (Presente:Ausente) & $4: 6$ & $6: 2$ & 0,19 & Test de Fisher \\
Índice T/B (mmHg) & $0,96 / 0,16$ & $0,870,10$ & 0,36 & Test de Wilcoxon \\
Vasculopatía (Normal:Moderada) & $6: 4$ & $5: 3$ & 1,00 & Test de Fisher \\
Germen detectado (TM:TR:Tsp) & $2: 5: 3$ & $1: 4: 3$ & 1,00 & Test de Fisher \\
Localización de la micosis (O:OP:P) & $8: 1: 1$ & $5: 1: 2$ & 0,76 & Test de Fisher \\
\hline
\end{tabular}

Para el caso de la distribución de sexos, se presenta la razón Hombres: Mujeres. Las distribuciones de variables continuas (edad, duración de la enfermedad, IMC) se compararon utilizando test de Student o de Wilcoxon. Para las variables dicotómicas, se utilizaron las pruebas de Chi cuadrado o Fisher ${ }^{(29)}$. IMC: índice de masa corporal; HbA1c: hemoglobina glicosilada, T/B: índice tobillo brazo; TM: T. mentagrophytes; TR: T. rubrum; Tsp: Trichophyton spp; O: onicomicosis; OP: onicomicosis y micosis plantar; P: micosis plantar.

test, $\mathrm{p}$-value $=0,19)$ a mayores tiempos de remisión en el grupo control (figura 1).

Destacamos que en los tres análisis se observó un efecto protector del tratamiento: mayor probabilidad de remisión en el grupo tratado en comparación con el grupo control.

No hubo eventos adversos en ninguno de los grupos y no hubo complicaciones relacionadas con la aplicación de las medias con NP Ag.

\section{Discusión}

Aunque las infecciones por tiña son mínimamente sintomáticas en pacientes no diabéticos, su frecuencia es alta y puede complicar al pie del diabético formando puntos de entrada que conducen a infecciones bacterianas graves ${ }^{(32)}$. Nuestros resultados, al igual que otros informes, sugieren que las especies de Trichophyton son los aislados fúngicos más comunes de las dermatomicosis del pie. Onicomicosis fue la infección más frecuente, seguida de la tiña pedis. Existe evidencia que sugiere que estas lesiones son factores de riesgo significativos para el desarrollo de celulitis de las extremidades inferiores $^{(8)}$. Esto puede prevenirse con una terapia farmacológica efectiva, pero requiere de tratamientos prolon$\operatorname{gados}^{(11)}$. En onicomicosis los tratamientos tópicos son efectivos si se usan solos para la afectación ungueal leve a moderada. Específicamente la onicomicosis blanca superficial restringida al dorso de la placa un-

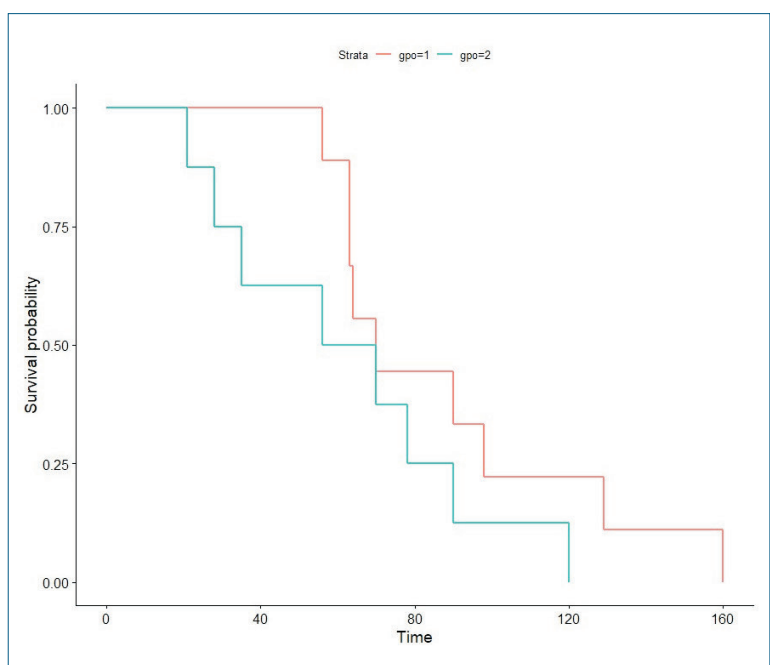

Figura 1. Gráfico de Kaplan-Meier. Tiempo a la remisión clínica en los 90 días. Menor tiempo para la población con NP Ag y mayores tiempos de remisión en I grupo de tratamiento convencional (Log-Rank test, p-value=0,19). Grupo 1: control. Grupo 2: intervención.

gueal y la onicomicosis subungueal lateral distal moderada. El tratamiento mecánico es efectivo como complemento de la terapia tópica incluyendo la avulsión y abrasión de las uñas, en especial en la onicomicosis subungueal parcial. Todos nuestros pacientes recibieron 


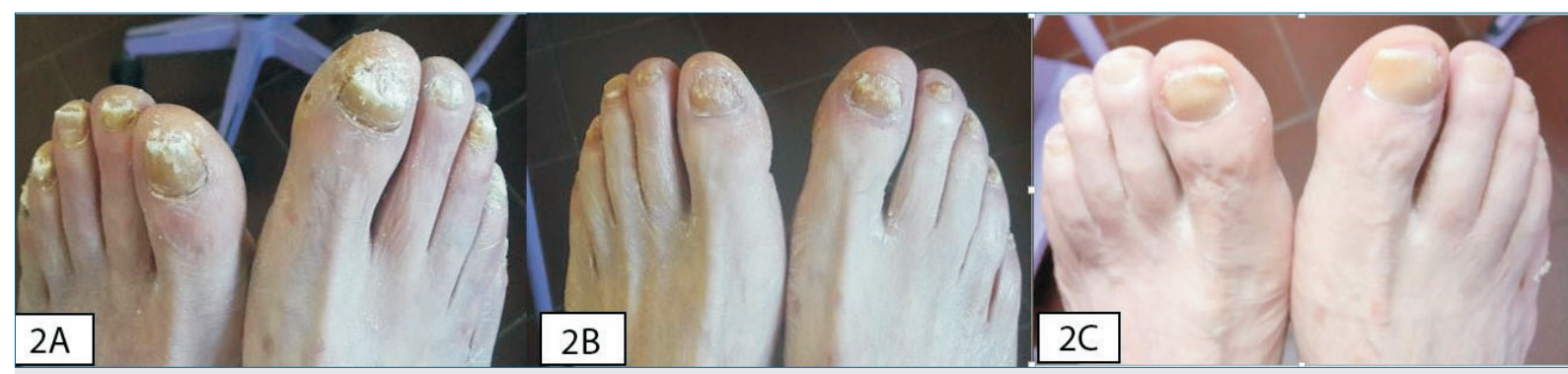

Figura 2. Imagen representativa de paciente con NPAg. Hombre, 70 años. 2A) Onicomicosis subungueal distal y lateral, destrucción de la lámina ungueal. 2B) Semana 2 de tratamiento. 2C) Semana 12: resolución clínica y microbiológica.

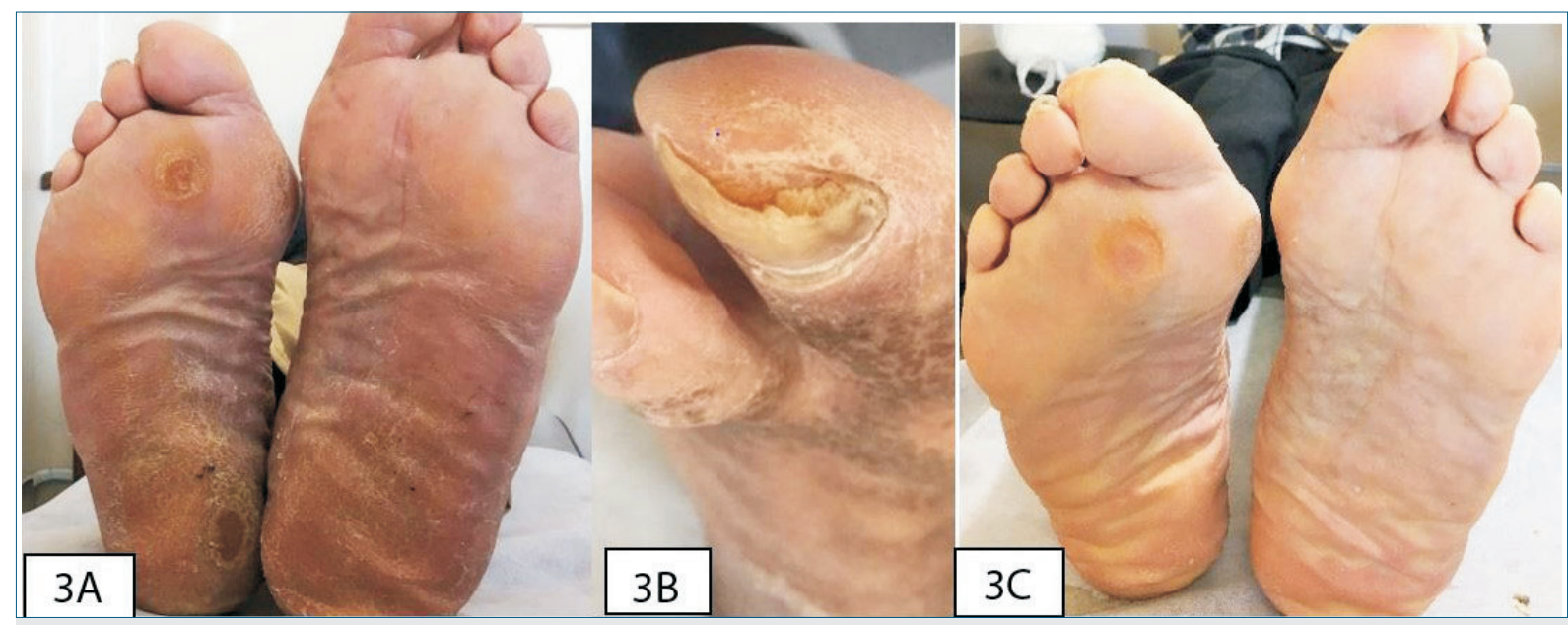

Figura 3. Imagen representativa de paciente con NP Ag. Hombre, 76 años. 3A) Tiña pedis, hiperqueratósica. 3B) Onicomisosis distal lateral. 3C) Semana 5 de tratamiento. Remisión clínica, microbiológica.

tratamiento tópico con terbinafina junto al tratamiento adyuvante mecánico. Sin embargo, una desventaja de los antifúngicos es la citotoxicidad sobre las células del mamífero y su naturaleza química poco soluble que afecta su biodisponibilidad provocando efectos adversos, fracasos terapéuticos y la aparición de resistencia con altas tasas de recaída y reinfección.

En el análisis comparativo los pacientes tratados con medias de NP Ag (frente a los controles) mostraron mayor tasa de curación y en un tiempo menor que el grupo control (figuras 2, 3 y 4).

Aunque las diferencias no fueron significativas, observamos una tendencia clara de mayor remisión en el grupo con NP Ag para las variables dicotómicas remisión clínica, remisión microbiológica, al igual que cuando evaluamos el tiempo a la remisión clínica, con una tendencia no significativa (figura 1) con mayores tiempos de remisión en el grupo control. Se destaca el resultado concordante en las tres evaluaciones.

La plata es un agente microbicida de amplio espectro. Su mecanismo de acción antifúngico es sobre la membrana del hongo modificando su permeabilidad o la capacidad respiratoria ocasionando la muerte celular. Las NP Ag liberan iones de plata que interactúan con los grupos tiol de muchas enzimas inactivándolas, o bien generando radicales libres que son extremadamente reactivos y presentan gran actividad citotóxi$\mathrm{ca}^{(33)}$. Se ha demostrado su acción citotóxica sobre Trichophyton mentagrophytes con respuestas comparables con anfotericina B y respuestas aun mayores cuando se comparan contra el fluconazol ${ }^{(34)}$. Otros nanoproductos han demostrado capacidad antifúngica contra $T$. rubrum $^{(35)}$. Un estudio reciente exploró el uso de nanopartículas como vehículo para ketoconazol en forma intradérmica controlada y sostenida en micosis superficial ${ }^{(36)}$.

En este textil la aplicación del nanoproducto a la molécula mediante técnica de electropresión permite una mayor resistencia al lavado. Su liberación lenta y sostenida durante más tiempo es diferente a las técnicas de inmersión o difusión de otros productos textiles, lo que empodera el efecto de las NP Ag. 


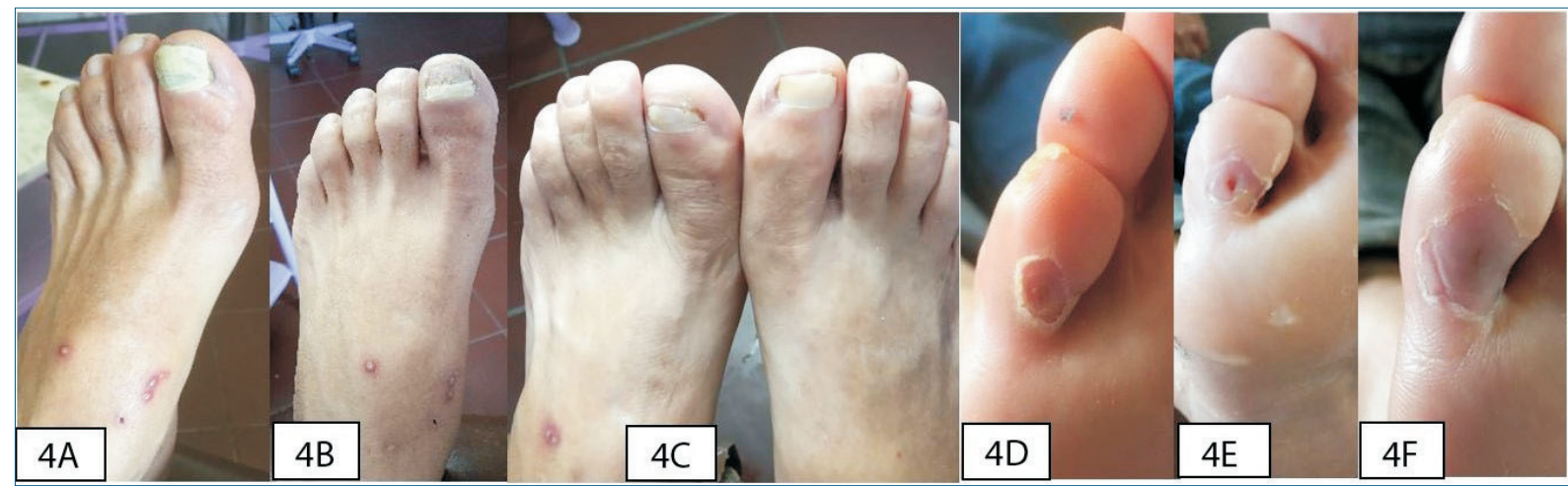

Figura 4. Imagen representativa de paciente con NP Ag. Mujer, 69 años. 4A-4B) Onicomicosis blanca superficial. 4A-4D) Lesiones estafilocócicas. Tratamiento local, mupirocina, terbinafina, resección parcial de la uña, NP Ag. 4C-4F) Semana 7: remisión clínica y microbiológica.

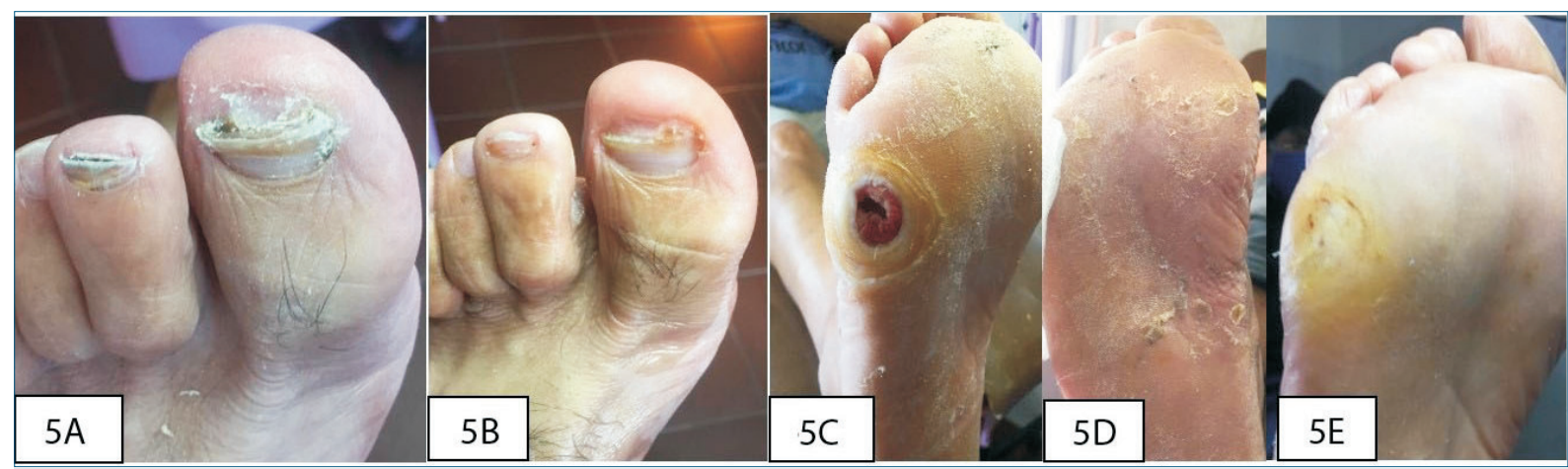

Figura 5. Imagen representativa de paciente con NP Ag. Hombre, 49 años. 5A) Onicomicosis distal y lateral. 5C-5D) Dermatofitosis plantar hiperqueratósica. Úlcera crónica neuropática en quinto metatarsiano. 5B-5E) Semana 4: remisión clínica y microbiológica. Úlcera epitelizada.

Una observación que destacamos en el grupo intervención fue la remisión de úlceras crónicas clínicamente no infectadas asociadas a la dermatomicosis (figuras $5 \mathrm{y}$ 6). Cuando se produce una herida, su entorno puede promover la formación de biopelículas de múltiples especies entre las bacterias y los hongos, con implicancias para la patogenicidad, el tratamiento y los resultados de la lesión, siendo este un momento óptimo para la intervención terapéutica ${ }^{(37)}$. Pensamos que el tratamiento estándar realizado a la úlcera (desbridado), sumado a la actividad biocida de las NP Ag sobre la superficie del pie, contribuye a modificar el microbioma impidiendo la formación de biopelículas que alteran la respuesta inmune y la cicatrización, hechos que deberán ser demostrados en una investigación futura.

No registramos efectos colaterales relacionados con el uso del textil con NP Ag. Otros estudios han demostrado que la toxicidad de NP Ag es mucho menor que la carga de masa equivalente de sales de plata ${ }^{(38)}$.

\section{Conclusión}

Nuestro estudio piloto muestra que el uso del textil confeccionado con hilos de NP Ag se asoció con una mayor probabilidad de curación completa en un período de 12 semanas en comparación con un tiempo mayor para el tratamiento habitual. Se pone de manifiesto el efecto positivo de NP Ag en el tratamiento de la dermato y onicomicosis a pesar de que el número de pacientes no nos permitió llegar al nivel de significación estadística.

Como ya se ha señalado, la prevención de la UPD representa la estrategia costo/efectiva con mejor resultado. Preservando el tejido sano, se evitan complicaciones asociadas a la aparición de una úlcera suplementaria.

Finalmente, luego de esta evaluación inicial, continuamos con las pruebas clínicas y el monitoreo de seguimiento para confirmar el valor de estas NP Ag en el tratamiento de las dermatomicosis.

Está en curso la inclusión de pacientes con úlceras crónicas con el objetivo de observar el resultado de la le- 


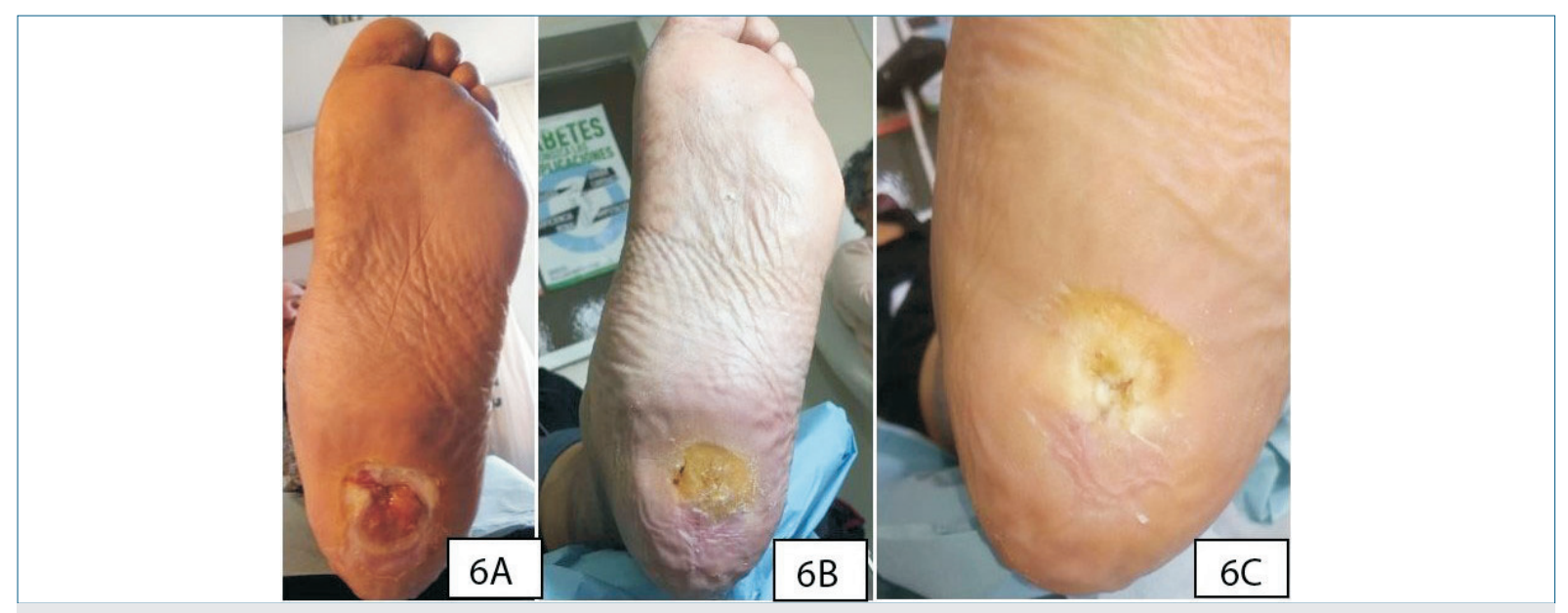

Figura 6. Imagen representativa de paciente con NP Ag. Hombre, 59 años. IRC. 6A) Micosis interdigital. Úlcera crónica de tres años de evolución. 6B) Semana 6. 6C) Semana 8: remisión clínica. Úlcera epitelizada.

sión y evaluar las implicancias de las NP Ag en la carga bacteriana de éstas.

\section{Declaración ética}

Todos los procedimientos realizados en este estudio están de acuerdo con los estándares éticos del comité de investigación institucional o nacional y con la Declaración de Helsinki de 1964 y sus enmiendas posteriores o estándares éticos comparables.

\section{Conflicto de interés}

La empresa Prumer Ltda. aportó el textil funcional (medias con NP Ag). Los autores declaran no tener ningún tipo de relación financiera con la empresa.

\section{Summary}

Onychomycosis and tinea pedis represent a significant proportion of infections in the diabetic foot, a common foot problem, and they constitute a threat to the viability of tissues that may provoke secondary bacterial infections. To combat them, antifungal treatments are required for long periods of time, the rates of relapse and reinfection being high. Several studies have proved the safety and effectiveness of silver nano particles (NP $\mathrm{Ag}$ ) as an antimicrobial agent.

A study was conducted to assess nanoparticle agents for foot dermatomycosis in diabetic patients.

Method: pilot, open, prospective randomized and controlled study in patients who are assisted in a diabetic foot policlinic. 18 patients complied with the inclusion criteria and two homogeneous groups were formed.

Both groups received standard treatment consisting in topic antifungal and mechanical roughing. The intervention groups used a textile (stockings) made with sil- ver nanoparticle threads. Clinical and microbiological control was made during 12 weeks, also assessing the remission percentage and the time it took to achieve it.

Resultados: onychomycosis and trichophyton rubrum prevailed. The intervention group showed a greater percentage of remission of lesions in a period of time that was shorter than that of the control group.

Conclusions: the use of stockings made with NP Ag threads was associated with a greater probability of complete healing, in a 12-week period, despite the fact that the number of patients was not statistically significant. This could contribute to the prevention of supplementary infections or ulcers in the diabetic foot.

\section{Resumo}

Uma proporção significativa de infecções do pé diabético consiste em onicomicose e Tinea pedis, um problema comum nos pés que ameaça a viabilidade do tecido e pode causar infecções bacterianas secundárias. Requerem períodos prolongados de tratamento antifúngico com altas taxas de recidiva e reinfecção. Vários estudos têm demonstrado a segurança e a eficácia das nanopartículas de prata (NP Ag) como agente antimicrobiano. Realizamos um estudo onde o tratamento com NP Ag foi avaliado na dermatomicose do pé de pacientes diabéticos.

Método: estudo piloto, aberto, prospectivo, randomizado e controlado em pacientes atendidos em uma policlínica de pé diabético. Dezoito pacientes preencheram os critérios de inclusão, formando dois grupos homogêneos. Ambos os grupos receberam tratamento padrão que consiste em antifúngico tópico e moagem mecânica. $\mathrm{O}$ grupo intervenção utilizou um tecido (meias) confeccionado com fios NP Ag. O controle clí- 
nico e microbiológico foi realizado até 12 semanas. A porcentagem de remissão e o tempo para alcançá-la foram avaliados.

Resultados: predominaram a onicomicose e o germe Trichophyton rubrum. No grupo intervenção, obteve-se maior percentual de remissão das lesões e em menor tempo que o grupo controle.

Conclusões: o uso de meias confeccionadas com fios NP Ag esteve associado a uma maior probabilidade de cura completa, no período de 12 semanas, apesar do número de pacientes não permitir atingir o nível de significância estatística, podendo contribuir para a prevenção de infecções ou úlceras adicionais no pé diabético.

\section{Bibliografía}

1. Lavery L, Davis K, Berriman S, Braun L, Nichols A, Kim P, et al. WHS guidelines update: Diabetic foot ulcer treatment guidelines. Wound Repair Regen 2016; 24(1):112-26.

2. Sandoya E. Diabetes y enfermedad cardiovascular en Uruguay. Rev Urug Cardiol 2016; 31(3):505-14.

3. Boulton A. The diabetic foot: a global view. Diabetes Metab Res Rev 2000; 16(Suppl 1):S2-5.

4. Lim H, Collins S, Resneck JJr, Bolognia J, Hodge J, Rohrer T, et al. The burden of skin disease in the United States. J Am Acad Dermatol; 76(5):958-972.e2. doi: 10.1016/j. jaad.2016.12.043

5. Jupiter D, Thorud J, Buckley C, Shibuya N. The impact of foot ulceration and amputation on mortality in diabetic patients. I: From ulceration to death, a systematic review. Int Wound J 2016; 13(5):892-903.

6. Spichler A, Hurwitz B, Armstrong D, Lipsky B. Microbiology of diabetic foot infections: from Louis Pasteur to 'crime scene investigation'. BMC Med 2015; 13:2. doi: 10.1186/ s12916-014-0232-0

7. Parada H, Veríssimo C, Brandão J, Nunes B, Boavida J, Duarte R, et al. Dermatomycosis in lower limbs of diabetic patients followed by podiatry consultation. Rev Iberoam Micol 2013; 30(2):103-8. doi: 10.1016/j.riam.2012.09.007

8. Roujeau J, Sigurgeirsson B, Korting H, Kerl H, Paul C. Chronic dermatomycoses of the foot as risk factors for acute bacterial cellulitis of the leg: a case-control study. Dermatology 2004; 209(4):301-7. doi: 10.1159/000080853

9. Bonasse J, Asconegui F, Conti-Díaz I. Estado actual de las dermatofitosis en el Uruguay. Rev Arg Micol 1982; 5(2):29-31

10. Öztürk A, Tapbakan M, Metin D, Yener C, Uysal S, Yýldýrým Pýmpýr I, et al. A neglected causative agent in diabetic foot infection: a retrospective evaluation of 13 patients with fungal etiology. Turk J Med Sci 2019; 49(1):81-6. doi: 10.3906/sag-1809-74

11. Baran R, Hay R, Garduno J. Review of antifungal therapy and the severity index for assessing onychomycosis: part I. J Dermatolog Treat 2008; 19(2):72-81. doi: 10.1080/09546630701243418
12. Ge L, Li Q, Wang M, Ouyang J, Li X, Xing M. Nanosilver particles in medical applications: synthesis, performance, and toxicity. Int $\mathrm{J}$ Nanomedicine 2014; 9:2399-407. doi: 10.2147/IJN.S55015

13. McNeil S. Nanotechnology for the biologist. J Leukoc Biol 2005; 78(3):585-94. doi: 10.1189/jlb.0205074

14. Morones J, Elechiguerra J, Camacho A, Holt K, Kouri J, Ramírez J, et al. The bactericidal effect of silver nanoparticles. Nanotechnology 2005; 16(10):2346-53. doi: 10.1088/0957-4484/16/10/059

15. Chopra I. The increasing use of silver-based products as antimicrobial agents: a useful development or a cause for concern? J Antimicrob Chemother 2007; 59(4):587-90. doi: 10.1093/jac/dkm006

16. Drake P, Hazelwood K. Exposure-related health effects of silver and silver compounds: a review. Ann Occup Hyg 2005; 49(7):575-85.

17. Kiwi J, Pulgarin C. Innovative self-cleaning and bactericide textiles. Catal Today 2010; 151(1-2):2-7.

18. Joshi M, Bhattacharyya A, Ali S. Characterization techniques for nanotechnology applications in textiles. Indian J Fibre Text Res 2008; 33(3):304-17.

19. Potiyaraji P, Kumlangdudsana P, Dubas S. Synthesis of silver chloride nanocrystal on silk fibers. Mater Lett 2007; 61(11-12):2464-6.

20. Toray Textiles Europe Limited. See it SAFE®. Mansfield, UK: Toray, 2020. Disponible en: http://www.ttel. co.uk/TTELSeeItSafe.html. [Consulta: 2020].

21. Zhang F, Wu X, Chen Y, Lin H. Application of silver nanoparticles to cotton fabric as an antibacterial textile finish. Fibers Polym 2009; 10(4):496-501.

22. MacKeen P, Person S, Warner S, Snipes W, Stevens SJr. Silver-coated nylon fiber as an antibacterial agent. Antimicrob Agents Chemother 1987; 31(1):93-9.

23. Radetice M. Functionalization of textile materials with silver nanoparticles. J Mater Sci 2013; 48:95-107. doi: 10.1007/s10853-012-6677-7

24. Hilgenberg B, Prange A, Vossebein L. Testing and regulation of antimicrobial textiles. En: Sun G, ed. Antimicrobial textiles. Cambridge: Elsevier, 2016:7-18.

25. Feldstein S, Totri C, Friedlander S. Antifungal therapy for onychomycosis in children. Clin Dermatol 2015; 33(3): 333-9.

26. Uruguay. Universidad de la República. Facultad de Medicina. Hospital de Clínicas. Repartición Microbiología. Departamento de Laboratorio Clínico. Manual de toma de muestras para estudio bacteriológico, parasitológico y micológico: selección, recolección, conservación y transporte. Montevideo: Hospital de Clínicas, 2004. Disponible en: http://ops-uruguay.bvsalud.org/pdf/laboratorio.pdf. [Consulta: 2020].

27. Carson J. Wound/abscess and soft tissue cultures: aerobic bacteriology. En: Leber A. Clinical microbiology procedures handbook. Washington, DC: ASM Press, 2016:Section 3.13.1.1-3.13.1.20. 
28. R Core Team. R: A language and environment for statistical computing. Vienna, Austria: R Foundation for Statistical Computing, 2019.

29. Agresti A. An introduction to categorical data analysis. 2 ed. New Jersey: John Wiley \& Sons, 2007.

30. Hosmer D, Lemeshow S, May S. Applied survival analysis. regression modeling of time to event data. New Jersey: John Wiley \& Sons, 1999.

31. Therneau T. A package for survival analysis in s. version 2.38. Vienna: Institute for Statistics and Mathematics, 2015. Disponible en: https://cran.r-project.org/web/packages/survival/index.html. [Consulta: 2020].

32. Akkus G, Evran M, Gungor D, Karakas M, Sert M, Tetiker T. Tinea pedis and onychomycosis frequency in diabetes mellitus patients and diabetic foot ulcers. A cross sectional observational study. Pak J Med Sci 2016; 32(4):891-5. doi: $10.12669 /$ pjms.324.10027

33. Prabhu S, Poulose E. Silver nanoparticles: mechanism of antimicrobial action, synthesis, medical applications, and toxicity effects. Int Nano Lett 2012; 2:32. doi: 10.1186/ $2228-5326-2-32$
34. Kim K, Sung W, Moon S, Choi J, Kim J, Lee D. Antifungal effect of silver nanoparticles on dermatophytes. J Microbiol Biotechnol 2008; 18(8):1482-4.

35. Flores F, de Lima J, Ribeiro R, Alves S, Rolim C, Beck R, et al. Antifungal activity of nanocapsule suspensions containing tea tree oil on the growth of Trichophyton rubrum Mycopathologia 2013; 175(3-4):281-6.

36. Wang F, Yang P, Choi J, Antovski P, Zhu Y, Xu X, et al. Cross-linked fluorescent supramolecular nanoparticles for intradermal controlled release of antifungal drug-a therapeutic approach for onychomycosis. ACS Nano 2018; 12(7): 6851-9. doi: 10.1021/acsnano.8b02099

37. Kalan L, Brennan M. The role of the microbiome in nonhealing diabetic wounds. Ann N Y Acad Sci 2019; 1435(1):79-92. doi: 10.1111/nyas.13926

38. Li W, Xie X, Shi Q, Zeng H, Ou-Yang Y, Chen Y. Antibacterial activity and mechanism of silver nanoparticles on Escherichia coli. Appl Microbiol Biotechnol 2010; 85(4):1115-22. doi: 10.1007/s00253-009-2159-5

\section{Contribución de autores}

Pablo Orellano, https://orcid.org/0000-0002-5080-5961. Concepción, diseño, ejecución, interpretación de los resultados, redacción. María del Huerto Urdangarin, https://orcid.org/0000-0002-5713-3352. Ejecución, recolección de datos, revisión.

Jenifer Silva, https://orcid.org/0000-0001-7118-8280. Concepción, diseño, revisión crítica.

Germán Botto, https://orcid.org/0000-0002-4055-9277. Diseño, análisis de datos, análisis de resultados, revisión crítica.

Analía González, https://orcid.org/0000-0001-8324-1835. Ejecución, recolección de datos, revisión.

Lilián Vucovich, https://orcid.org/0000-0003-0143-4524. Ejecución, revisión crítica.

Vivian Peirano, https://orcid.org/0000-0001-8839-8495. Ejecución, análisis de muestras microbiológicas, revisión. 